\title{
Systematic management approach to pharmacotherapy for people with learning disabilities
}

\author{
Stewart L. Einfeld
}

With progressive deinstitutionalisation in many countries, the general psychiatrist is now more frequently requested to provide assistance with behavioural problems in people with learning disabilities. There are a range of challenges in providing quality care for these people. There are challenges in assessment and diagnosis, in coordinating behavioural and social interventions with pharmacotherapy, and in the lack of psychopharmacological research in this population, which results in a diminished evidence base. In addition, there are numerous factors beyond the patient. These include legal consent requirements and the complex system of carers surrounding those with disabilities with whom the practitioner may need to interact.

Behavioural and emotional difficulties have consistently been shown to be two to three times more common in persons with learning disabilities than in the rest of the population, whichever assessment method has been used (Corbett, 1979; Richardson et al, 1979; Einfeld \& Tonge, 1995). Behavioural and emotional problems are rated by parents as the major source of stress in caring for a child with a learning disability and they are the major reasons for failure of employment and reinstitutionalisation (Corbett, 1979). They greatly increase the costs of care provision by governments or private agencies. Currently there is major concern in many countries about the occupational health and safety costs arising from injuries to care workers caused by clients' disturbed behaviour.

These worries can lead to strident and sometimes unrealistic demands for the psychiatrist to solve the problem by prescribing medication in adequate quantity to 'control' the situation. At the same time, however, there has been concern about the overprescription of medication to persons with learning disabilities, particularly excessive use of antipsychotics with considerable side-effects. This has sometimes led to inappropriately restrictive attitudes towards medication use. In some regions, authorities have been set up to authorise or monitor prescribing of medications, especially psychotropic drugs, to persons with learning disabilities. These authorities vary in their sophistication, understanding, and cooperation with - or hostility towards - medical practitioners. Thus, the psychiatrist is often caught in the bind of pressure from some quarters to prescribe to relieve a desperate situation and objections to such practice from others.

\section{Diagnostic and assessment issues}

A number of papers and texts discuss diagnostic and assessment issues in dealing with the psychiatric problems of people with learning disabilities (Einfeld, 1992; Santosh \& Baird,1999). A couple of common challenges deserve particular mention.

First is the diagnosis of psychosis. The psychiatrist is often told by carers that a patient is hallucinating. The evidence proposed for this is that the person has been seen talking to herself or himself,

Stewart L. Einfeld is Associate Professor of Psychiatry at the University of New South Wales, Sydney (School of Psychiatry, UNSW, 2 Short Street, Kogarah, 2217 NSW, Australia; e-mail: s.einfeld@unsw.edu.au). He is also Director of Child and Adolescent Psychiatry Programs for South East Sydney Area Health Service. Academic interests are in the psychiatry of learning disability, especially behaviour phenotypes of genetic disorders and taxonomy and measurement of psychopathology. 
either alone or in the company of others. It is much more likely that such behaviours are due to limitations in social skills than to hallucinations. Usually, the person is remembering or fantasising about a conversation, although doing so aloud. Similarly, grandiose ideas are more likely to be an attempt to compensate for poor self-esteem than to be true delusions, since they are not really believed by the person expressing them. To clarify the nature of these behaviours, there is no alternative to spending enough time discussing these experiences with the patient. It is generally said that psychosis cannot be reliably diagnosed below an IQ of about 45 , although this has not been critically determined.

If the psychiatrist cannot diagnose psychosis with confidence, this does not mean that antipsychotics cannot be prescribed. However, treatment should be aimed at particular target symptoms rather than at an uncertain syndrome.

Second, depression has perhaps been underdiagnosed in this population. This is understandable given that limited verbal skills may prevent people with learning disability from reporting their mood, thus denying psychiatrists assessment of the cardinal symptom of depression. Nevertheless, Evans et al (1999) have demonstrated that the behavioural symptoms of depression from DSM-IV (American Psychiatric Association, 1994) can be reliably rated by nurses even in persons with profound learning disability; and Clarke \& Gomez (1999) have shown that such patients diagnosed using modified depression criteria responded to antidepressant treatment. Thus, depression can be considered, and antidepressants tried, if a person with learning disability presents with episodic periods of withdrawal, loss of interest in usual activities, crying, looking miserable, diurnal behavioural changes, and appetite and sleep disturbance. These behaviours can be associated with other evidence of psychological dysfunction, such as an increase in selfinjury, decline in self-help skills, disruptive behaviours or anxiety. Although some writers have asserted that these latter behaviours can be depressive equivalents, the diagnosis is less likely than when the above behavioural aspects of depression are also present.

The lack of a valid and comprehensive taxonomy of psychiatric disorder is being addressed by efforts in the UK, Australia and the USA. Under the chairmanship of Professor Anne Cooper, The Royal College of Psychiatrists has produced a set of diagnostic criteria which will soon be available for evaluation (Royal College of Psychiatrists, 2001). The World Health Organization Division of Mental Health (1996) has issued an ICD-10 Guide for Mental Retardation, although this has many limitations (Einfeld $\&$ Tonge, 1999). The current uncertainty in diagnosis has one effect on prescribing: many behavioural and emotional problems need to be treated at the symptomatic rather than syndrome disorder level.

In order to pursue rational pharmacotherapy in the face of the above challenges, a number of authors have proposed systematic management approaches. One such is described below, adapted from Einfeld (1990).

\section{Guidelines for using psychotropic medication in people with learning disabilities}

The decision to prescribe psychotropic medication should follow a comprehensive assessment of the individual's emotional and behavioural disturbance. Such an assessment will include not only descriptions of the behaviour and the formulation of precipitating and palliating factors, but also an assessment of the efficacy of all previous modes of treatment. The psychiatrist should try to resist 'knee-jerk' responses, despite the pressure of crisis presentations.

Proper consideration should be given to the issue of informed consent, particularly with respect to legal requirements. Frequently, the practitioner is required to make an all-or-nothing decision about a person's capacity to consent. In fact, capacity will be quite variable. For example, a person with mild learning disability may be able to understand that an antipsychotic may make them feel calmer, but not weigh up the advantages of that against the risk of side-effects.

The psychotropic treatment needs to be an integrated part of other concurrent treatments. It is unusual for psychotropic medication alone to be sufficient. This requires good interdisciplinary communication.

The precise target symptoms for which psychotropic medication is being prescribed should be stated. In other words, what exactly is it hoped that this medication will treat? It is very difficult to gain reliable information from global impressions such as "patient will be less disruptive" or "will be more compliant". Better to use specific descriptions such as "patient will hit others less frequently".

One needs to establish some method for reliably and validly documenting changes in the target symptoms during the course of treatment. Such a system is shown in Figs 1 and 2. This record should demonstrate that target symptoms have had a positive response to the medication before it is continued. While this is obvious, it is common for patients to be seen who have been receiving large doses of antipsychotics for many years without any evidence of their benefit.

When target symptoms have been reduced or absent for a reasonable period, an attempt should 


\begin{tabular}{|l|l|l|l|l|l|l|l|l|}
\hline \multirow{2}{*}{ Behaviour } & \multicolumn{3}{|l|}{ Date } & \multicolumn{4}{l|}{$\begin{array}{l}\text { Weekly } \\
\text { total }\end{array}$} \\
\hline & & & & & & & & \\
\hline & & & & & & & & \\
\hline & & & & & & & & \\
\hline & & & & & & & & \\
\hline & & & & & & & & \\
\hline & & & & & & & & \\
\hline
\end{tabular}

Fig. 1 Behaviour monitoring chart. Each behaviour is scored daily ( $0=$ not a problem; $1=a$ moderate problem; $2=$ major problem), and the scores are then totalled for the week.

be made to reduce the dose. Again, reliable and valid measures of the effect of this dosage change should be available. Patients are frequently maintained on medication for lengthy periods long after symptoms have resolved, out of fear that the behaviours will recur, even when the patient's circumstances have changed.

When a psychotropic medication is withdrawn, a proper withdrawal regime should be designed. Withdrawal symptoms appear commonly in this population if long-standing treatments are terminated suddenly. A regime I might use for reducing antipsychotics is a reduction in daily dose of $20 \%$ per month.

The presence of organic brain dysfunction often results in idiosyncratic response to psychotropic drugs. This suggests that small doses should be used initially, with close attention paid to the emergence of side-effects.

Finally, general principles of pharmacotherapy must be observed as with any other patient; that is,

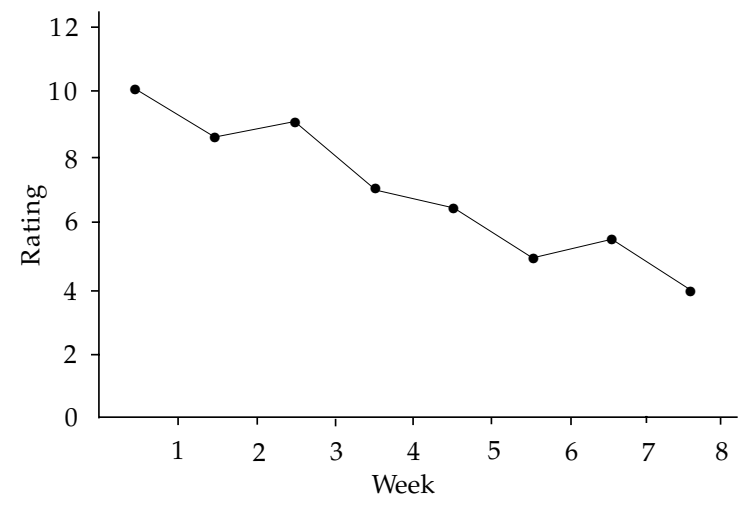

Fig. 2 Example of a chart summary proper attention should be paid to issues of compliance, pharmacokinetics, drug interactions and side-effects.

The chart shown in Fig. 1 has been found useful for monitoring behaviour. From this, a summary graph (Fig. 2) can be plotted, demonstrating change in the targeted behaviour (in this example, a progressive improvement). The use of such charts is necessary because prescribers will frequently be given conflicting information from various carers who attend consultations, each of whom will have a different experience of the patient and different interpretations of behaviours manifested. A chart can provide a far more objective account of behaviour than traditional incident records. It is best for the prescriber to ask a particular senior carer who will be observing the patient regularly to keep a chart record. It is also useful to have two other carers completing charts independently, to provide the basic measure of interrater reliability. Such charts may also be completed in other contexts, for example, by a carer in a group home and in an activity centre or by each parent of a child.

\section{Factors in prescribing particular medications}

\section{Antipsychotics}

Antipsychotics are the most researched medications in the learning disability field. Nevertheless, in a systematic review Brylewski \& Duggan (1999) found only three randomised controlled trials of a sufficient 
standard to include. These were inconclusive concerning the effects of antipsychotics on challenging behaviours. However, lower-level evidence suggests that antipsychotics reduce challenging behaviours, although with significant side-effects (Smith \& Perry, 1992).

Antipsychotic medications frequently cause sedation and compromise cognitive function and self-help skills. In addition, extrapyramidal sideeffects are common. Newer antipsychotic drugs, such as olanzapine and risperidone, have been widely introduced in clinical practice. Although these agents cause lower levels of short-term extrapyramidal sideeffects, there is only limited evidence that the risk of tardive dyskinesia is lessened (Beasley et al, 1999). Both of these medications commonly cause troublesome weight gain. They are also associated with raised liver enzymes (Benazzi, 1998), but the clinical significance of this is unclear. It should be appreciated that there is no substantive evidence that these newer antipsychotics have any greater efficacy than the traditional ones.

Buzan et al (1998) have reviewed 84 published cases of clozapine use in adults with learning disabilities. They concluded that clozapine was both efficacious and well tolerated. It is possible that the haematological side-effects of clozapine have led practitioners to be too cautious in using this medication.

Campbell et al (1997) found that 34\% of children with autism were receiving haloperidol over a prolonged period and developed dyskinesias, mostly associated with withdrawal.

Akathisia is an important side-effect of antipsychotics to consider in persons with learning disability. People with limited verbal skills are frequently unable to describe the sense of restlessness and irritability, which can be very distressing. This can lead to increased agitation, which may mistakenly be seen as an indication for increased doses of antipsychotics, making the problem worse. Carers are sometimes very surprised to see a decline in agitation or aggression when antipsychotic dose is reduced.

Another rare but important complication of antipsychotic medication in learning disability is neuroleptic malignant syndrome. Boyd (1993; cited in Reiss \& Aman, 1998) found the fatality rate (21\%) to be double that occurring in persons without learning disability.

\section{Antidepressants}

As stated, depression can be difficult to diagnose with confidence, owing to the patient's likely limited verbal skills. However, given the safety of selective serotonin reuptake inhibitors (SSRIs), a low threshold for instigation of an antidepressant trial is reasonable. The SSRIs have largely supplanted tricyclic antidepressants in the treatment of depression in this population, given the lesser impact of side-effects. Agitation and nausea are the most common. There is inconclusive evidence that one SSRI has any greater efficacy than another. As stated above, it is important to start with small doses, and for this reason SSRIs available in liquid form present advantages in that titration of small doses is possible.

The SSRIs have also been widely used in the field to treat perseverative and repetitive behaviours, including rituals of autism, preoccupations in Asperger's syndrome and self-injurious behaviour. From a retrospective case-note study Branford et al (1998) determined that about one-third of recipients showed improvement in these behaviours, although general maladaptive behaviours were also included. Collective professional evidence at least suggests that moderate improvements in stereotyped behaviours occur in at least half of recipients. Singh et al (1998) found marked reductions in self-injurious behaviour in 10 out of 12 individuals treated with fluoxetine or fluvoxamine.

\section{Anxiolytics}

There have been no specific studies of the treatment of anxiety in people with learning disability. Nevertheless, clinicians using SSRIs to treat phobic or generalised anxiety disorders and obsessivecompulsive disorder in learning disability report improvement similar to that seen in the general population. Moclobemide is an alternative, but in my experience it has less efficacy than the SSRIs. Buspirone has been described in case reports as of assistance, but there are no reliable studies demonstrating its effectiveness. There is little or no indication for the use of benzodiazapines for the treatment of behavioural and emotional disturbance, although they are commonly used in the treatment of epilepsy. Benzodiazapines, particularly clonazepam, frequently cause disinhibition and irritability in persons with organic brain impairments. When a patient with learning disability taking benzodiazepines presents with these symptoms, it is always worth asking the patient's neurologist to consider an alternative anticonvulsant.

Clonidine is used as an antihypertensive in adults, but it also has a sedative and calming effect, especially in children. The daily dose should be less than $5 \mu \mathrm{g} / \mathrm{kg}$ and blood pressure must be monitored by a competent person each time the dose is increased. The sedating effect tends to wear off after several months. If the dose is gradually reduced and stopped, and then reintroduced, the calming effect 
returns. Prescription of clonidine does require confidence that doses will not be omitted or the medication stopped suddenly, since hypertensive reactions can occur. Clonidine can also be useful in tic disorders and as an adjunct to treatment of attention-deficit hyperactivity disorder (ADHD).

\section{Mood stabilisers}

Medications included in this group are lithium, carbamazepine and valproate. These may be used either for the treatment of manic-depressive spectrum conditions, or for the treatment of disruptive behaviours. Typical manic-depressive illness is occasionally seen in learning disability, but more frequently patients present with what might be termed instability of mood, that is to say rapid alternations between excitement, irritability and high levels of activity, and withdrawal and loss of interest. There have been a number of studies of lithium treatment of disruptive behaviour in people with learning disability. For example, Langee (1990) found that $42 \%$ of individuals treated with lithium improved significantly. Lithium has also been widely used for impulsive, aggressive behaviour, although evidence for particular efficacy in this presentation is equivocal.

It should be noted that hypothyroidism is more common in Down's syndrome and that tremors and other movement disorders are more common in persons with learning disabilities, so particular care is needed to monitor for the side-effects of lithium in this population. Carbamazepine and valproate have been widely used as mood stabilisers in learning disability and generally have lower toxicity risks than lithium. There are surprisingly few studies of the use of this widely prescribed medication, and those that are published are predominantly case reports. Carbamazepine, of course, has the disadvantage of requiring more frequent monitoring of serum level, which SSRIs tend to raise. Valproate can cause liver disease, especially in younger children, and liver function should be monitored in the initial stages of its use.

\section{Stimulants}

Although stimulants have been tried for use in a range of behaviour problems associated with learning disability, their principal indication is for the same purpose as in children with learning disability: for the treatment of ADHD.

The diagnosis of ADHD is more complex in those with learning disability. It requires that the symptoms of inattention or hyperactivity and impulsivity are maladaptive and inconsistent with the developmental level. However, developmental level may not be determined accurately and it interacts with chronological age, which itself is associated with declining motor overactivity over time. There is some evidence to suggest that those with moderate and severe learning disability do not respond as well to stimulants as those with mild learning disability (Aman et al, 1991). It should be noted that stimulants may worsen epilepsy, anxiety and tics, all of which are more common in learning disability.

The SSRIs have fewer side-effects than stimulants for the treatment of ADHD, although they appear to be slightly less potent. As mentioned above, clonidine also has a calming effect in hyperactivity, although its costs are sedation, tolerance development and the need to monitor blood pressure.

I am not aware of any systematic studies of the use of stimulants in Prader-Willi syndrome, despite their appetite-suppressant properties.

\section{Anticonvulsants}

Anticonvulsants are mentioned here because they are frequently prescribed for persons with learning disabilities, either for management of epilepsy or as mood stabilisers. Psychiatrists should be aware of their psychotropic side-effects. Vigabatrin is used less commonly now because it causes visual field defects. It has also been observed to cause apparently psychotic behaviour. It can cause marked changes in alertness in persons with learning disability and severe epilepsy: it can both increase alertness and even improve cognitive function, but also apparently decrease alertness and awareness. Lamotrigine can also improve alertness, but may cause decline in cognitive function. It has also been noted occasionally to cause insomnia, irritability or aggression. Topiramate can improve or diminish alertness and cognitive function, sometimes quite markedly. In addition, it frequently causes nausea and anorexia.

\section{Antilibidinal agents}

The inappropriate sexual behaviour of some with learning disability can cause considerable concern and result in their social restriction. Consequently, there has been a growth in use of testosterone antagonists to reduce libido. Cyproterone is now widely favoured, as it is active orally, but it can be associated with weight gain. Luteinising-hormone releasing hormone (LHRH) analogues are the most effective and safest method for reducing testosterone levels over prolonged periods, but they are very expensive and of limited availability. The pharmacological reduction of libido is potentially of some 
ethical concern. Medication should be used as a last resort, only if efforts to redirect inappropriate sexual behaviour through behavioural and educational means have failed. In such cases suppression of this natural aspect of human function may be in the best interest of the patient. These issues are discussed in greater detail by Clarke (1989).

\section{Conclusion}

Behavioural and emotional problems are a major complication of learning disabilities and care providers greatly appreciate effective assistance in their control from psychiatric practitioners. The application of established assessment principles, a rational-empirical approach to drug trials and integration with other modes of treatment are of most value.

\section{References}

Aman, M. G., Marks, R. E., Turbott, S. H., et al (1991) Clinical effects of methylphenidate and thioridazine in intellectually subaverage children. Journal of the American Academy of Child and Adolescent Psychiatry, 30, 246-256.

American Psychiatric Association (1994) Diagnostic and Statistical Manual of Mental Disorders (4th edn) (DSM-IV). Washington, DC: APA.

Beasley, C., Dellva, M., Tamura, R., et al (1999) Randomised double-blind comparison of the incidence of tardive dyskinesia in patients with schizophrenia during long-term treatment with olanzapine or haloperidol. British Journal of Psychiatry, 174, 23-30.

Benazzi, F. (1998) Risperidone-induced hepatotoxicity. Pharmacopsychiatry, 31, 241.

Boyd, R. (1993) Antipsychotic malignant syndrome and mental retardation: review and analysis of 29 cases. American Journal of Mental Retardation, 98, 143-155 (erratum 98, 359).

Branford, D., Bhaumik, S. \& Naik, B. (1998) Selective serotonin re-uptake inhibitors for the treatment of perseverative and maladaptive behaviours of people with intellectual disability. Journal of Intellectual Disability Research, 42, 301-306.

Brylewski, J. \& Duggan, L. (1999) Antipsychotic medication for challenging behaviour in people with intellectual disability: a systematic review of randomized controlled trials. Journal of Intellectual Disability Research, 43, 360-371.

Buzan, R. D., Dubovsky, S. L., Firestone, D. et al (1998) Use of clozapine in 10 mentally retarded adults. Journal of Neuropsychiatry and Clinical Neurosciences, 10, 93-95.

Campbell, M., Armenteros, J. L., Malone, R. P., et al (1997) Antipsychotic-related dyskinesias in autistic children: a prospective, longitudinal study. Journal of the American Academy of Child and Adolescent Psychiatry, 36, 835-843.

Clarke D. J. (1989) Antilibidinal drugs and mental retardation: a review. Medicine, Science and the Law, 29, 136-146.

— \& Gomez, G. A. (1999) Utility of modified DCR-10 criteria in the diagnosis of depression associated with intellectual disability. Journal of Intellectual Disability Research, 43, 413-420.

Corbett, J. A (1979) Psychiatric morbidity and mental retardation. In Psychiatric Illness and Mental Handicap (eds F. E. James \& R. P. Snaith), pp. 11-25. London: Gaskell.

Einfeld, S. L. (1990) Guidelines for the use of psychotropic medication in patients with intellectual handicaps.
Australian and New Zealand Journal of Developmental Disabilities, 16, 71-73.

- (1992) Clinical assessment of psychiatric symptoms in mentally retarded individuals. Australian and New Zealand Journal of Psychiatry, 26, 48-63.

- \& Tonge, B. J. (1995) The Developmental Behavior Checklist: the development and validation of an instrument to assess behavioral and emotional disturbance in children and adolescents with mental retardation. Journal of Autism and Developmental Disorders, 25, 81-104.

— \& - (1999) Observations on the use of the ICD-10 guide for mental retardation. Journal of Intellectual Disability Research, 43, 408-412.

Evans, K., Cotton, R., Einfeld, S., et al (1999) The assessment of the behavioural criteria for depression in adults with severe or profound mental retardation. Journal of Intellectual and Developmental Disability, 24, 147-160.

Langee, H. R. (1990) Retrospective study of lithium use for institutionalized mentally retarded individuals with behaviour disorders. American Journal on Mental Retardation, 94, 448-452.

Reiss, S. \& Aman, M. (eds) (1998) Psychotropic Medication and Developmental Disabilities: The International Consensus Handbook. Columbus, $\mathrm{OH}$ : Ohio State University Nisonger Center.

Richardson, S. A., Katz, M., Koller, H., et al (1979) Some characteristics of a population of mentally retarded young adults in a British city. A basis for estimating service needs. Journal of Mental Deficiency Research, 25, 253-256.

Royal College of Psychiatrists (2001) DC-LD: Diagnostic Criteria for Psychiatric Disorders for Use with Adults with Learning Disabilities/Mental Retardation. Occasional Paper OP48. London: Gaskell.

Singh, A., Kleynhans, D. \& Barton, G. (1998) Selective serotonin re-uptake inhibitors in the treatment of selfinjurious behaviour in adults with mental retardation. Human Psychopharmacology, 13, 267-270.

Santosh, P. J. \& Baird, G. (1999) Psychopharmacotherapy in children and adults with intellectual disability. Lancet, 354 233-242

Smith, D. A. \& Perry, P. J. (1992) Nonantipsychotic treatment of disruptive behaviour in organic mental syndromes. Annals of Pharmacotherapy, 26, 1400-1408.

Whitworth, A. B., Liensberger, D. \& Fleischhacker, W. W (1999) Transient increase of liver enzymes induced by risperidone: two case reports. Journal of Clinical Psychopharmacology, 19, 475-476.

World Health Organization Division of Mental Health (1996) ICD-10 Guide for Mental Retardation. Geneva: WHO.

\section{Multiple choice questions}

1. Carers' reports that a person with a learning disability is "hallucinating" probably describe:

a poor socialisation

b fantasies

c psychotic perceptual disturbances

d memories spoken out loud.

2. Depression in people with learning disabilities:

a cannot be assessed reliably in those with severe disability

$\mathrm{b}$ responds to antidepressants only when the patient is able to describe his/her mood

c can be considered a valid diagnosis on the basis of behavioural signs alone

$\mathrm{d}$ can be reliably diagnosed from the presence of depressive equivalent behaviours alone. 
3. When prescribing psychotropic medication for persons with learning disabilities:

a disorders, not symptoms, should be the target of therapy

b verbal reports of several carers should be used to monitor treatment

c dosage levels used in the general population are a good guide for this population

$\mathrm{d}$ compliance is generally not a problem, as the medication is offered by carers or parents.

4. With respect to psychotropic medications in persons with learning disability:

a olanzapine and risperidone are more effective than traditional antipsychotics

b weight gain with olanzapine and risperidone is less of a problem than with traditional antipsychotics

c neuroleptic malignant syndrome is more common in people with learning disabilities

$\mathrm{d}$ benzodiazapines are a safe and effective alternative to antipsychotics.
5. With respect to psychotropic medications in persons with learning disabilities:

a in controlled trials, SSRIs were effective for the treatment of anxiety

b SSRIs are useful for the management of autistic rituals and insistence on sameness

c dexamphetamine's appetite-suppressant properties make it effective in treating Prader-Willi syndrome

d cyproterone has no significant side-effects.
MCQ answers

$\begin{array}{llllll}1 & 2 & 3 & 4 & 5\end{array}$

a $T$ a $\quad$ a $F$ a $F$ a $F$ a $F$

b $\begin{array}{llllllll}\text { T } & \text { b } & \text { b F } & \text { b F } & \text { b } T\end{array}$

c $F$ c $T$ c $F$ c $T$ c $F$

d $\begin{array}{llllll}\text { T } & \text { d F } & \text { d F } & \text { d F } & \text { d F }\end{array}$

\section{Mental Health Outcome Measures Second Edition Edited by Michele Tansella and Graham Thornicroft}

The importance of assessing outcome in mental health services has never been greater. The principles of evidence-based medicine demand that psychiatric outcomes are measurable and practicable. Yet in many areas of psychiatric practice there are gaps in the evidence and outcomes are notoriously difficult to quantify.

This multi-author text, revised and updated for the new decade, guides the reader through the minefield of mental health outcome measurement. Containing up-to-the minute reviews by the world-renowned experts in the field, Mental Health Outcome Measures equips the reader with the building blocks for evidence-based mental health service provision and evaluation.

This book will be of interest to clinicians and researchers in mental health and related fields, and to all those involved in researching, purchasing and delivering mental health services.

March 2001, £25.00, paperback, ISBN 1901242552

Book Sales, The Royal College of Psychiatrists, 17 Belgrave Square, London SW1X 8PG.

Tel: +44 (0)20 72352351 ext. 146; Fax: +44 (0)20 72451231

The latest information on College publications is on the Internet at www.rcpsych.ac.uk 